\title{
Stochastic Stability Analysis of Fuzzy Hopfield Neural Networks With Time-Varying Delays
}

\author{
He Huang, Daniel W. C. Ho, and James Lam
}

\begin{abstract}
The ordinary Takagi-Sugeno (TS) fuzzy models have provided an approach to represent complex nonlinear systems to a set of linear sub-models by using fuzzy sets and fuzzy reasoning. In this paper, stochastic fuzzy Hopfield neural networks with timevarying delays (SFVDHNNs) are studied. The model of SFVDHNN is first established as a modified TS fuzzy model in which the consequent parts are composed of a set of stochastic Hopfield neural networks with time-varying delays. Secondly, the global exponential stability in the mean square for SFVDHNN is studied by using the Lyapunov-Krasovskii approach. Stability criterion is derived in terms of linear matrix inequalities (LMIs), which can be effectively solved by some standard numerical packages.
\end{abstract}

Index Terms-Fuzzy systems, Hopfield neural networks, stability, stochastic systems, time-varying delay systems.

\section{INTRODUCTION}

$\mathbf{T}$ HE SO-CALLED Hopfield neural networks were first introduced by Hopfield [8]. For a few decades, Hopfield neural networks have been extensively investigated. Many applications have been found in different fields such as combinatorial optimization, signal processing and pattern recognition, see for examples [8], [9], [12], [13], [19]. These applications are built upon the stability of the equilibrium of neural networks. Thus, the stability analysis is a necessary step for the design and applications of neural networks. Sometimes, neural networks have to be designed such that there is only one equilibrium and this equilibrium is globally stable. For example, when a neural network is applied to solve the optimization problem, it must have one unique equilibrium which is globally stable. On the other hand, both in biological and artificial neural networks, the interactions between neurons are generally asynchronous which inevitably result in time delays. In electronic implementation of analog neural networks, nevertheless, the delays are usually time-varying due to the finite switching speed of amplifiers. It is known that time delays are often a source of instability of neural networks [16]. Therefore, it is of great importance to study the global stability of neural networks with time-varying delays.

Manuscript received February 4, 2004; revised August 17, 2004. This work was supported in part by the Research Grants Council under Grant CityU 101103, Grant HKU 7102/01P and by the Natural Science Foundation, Jiangsu Province, China, under Grant BK2003001. This paper was recommended by Associate Editor T. Saito.

H. Huang is with the Department of Computer Science and Engineering, Southeast University, Nanjing 210096, China, and also with the Department of Mathematics, City University of Hong Kong, Hong Kong (e-mail: h-huang@seu.edu.cn).

D. W. C. Ho is with the Department of Mathematics, City University of Hong Kong, Hong Kong (e-mail: madaniel@ cityu.edu.hk).

J. Lam is with the Department of Mechanical Engineering, University of Hong Kong, Hong Kong (e-mail: jlam@hku.hk).

Digital Object Identifier 10.1109/TCSII.2005.846305
When performing the computation, there are many stochastic perturbations that affect the stability of neural networks. It was pointed out [14], [15] that a neural network could be stabilized or destabilized by certain stochastic inputs. It implies that the stability analysis of stochastic neural networks also has primary significance in the research of neural networks. However, the stability analysis for stochastic neural networks is difficult. Recently, although the stability analysis of neural networks has received much attention, the stability of stochastic neural networks has not been widely studied. In [1], [2], [11], [14], some results related to this issue have been reported.

Fuzzy logic theory has shown to be an appealing and efficient approach to dealing with the analysis and synthesis problems for complex nonlinear systems. In [18], Takagi and Sugeno proposed an effective way to transform a nonlinear dynamic system to a set of linear sub-models via some fuzzy models by defining a linear input/output relationship as its consequence of individual plant rule. In Takagi-Sugeno (TS) fuzzy models, local dynamics in different state space regions is represented by linear models. The overall fuzzy model of the system is obtained by fuzzy "mixing" of these linear models. Then the analysis of the nonlinear system is based on these linear models. Moreover, in [4], the standard TS fuzzy model was extended to one with time delays, and some stability conditions were presented in terms of linear matrix inequalities (LMIs).

In this brief, we further extend the ordinary TS fuzzy models to describe the delayed Hopfield neural networks which are subjected to environmental noise. That is, the generalized TS fuzzy models can be used to represent some complex nonlinear systems by having a set of nonlinear stochastic delayed Hopfield neural networks as its consequent parts. This fuzzy modeling method is simple and natural. The system dynamics is captured by a set of fuzzy implications which characterize local relations in the state space. The local dynamics of each fuzzy rule is expressed by a stochastic Hopfield neural network with time-varying delay. The overall fuzzy model can be achieved by fuzzy "blending" of these nonlinear neural networks. To the best of our knowledge, this is the first time to introduce and study stochastic fuzzy Hopfield neural networks with time-varying delays, (SFVDHNNs). The main purposes of this paper are firstly to present the model of SFVDHNN by using fuzzy IF-THEN rules; and secondly to discuss the stability of SFVDHNN by constructing some appropriate Lyapunov-Krasovskii functional. One criterion is given to guarantee the global exponential stability in the mean square for SFVDHNN, which is formulated in terms of LMIs. It is known that LMIs can be efficiently solved [3], [7], [17]; therefore, our proposed result is practical. 
Notations: The following notations will be used throughout this paper. Let $\mathbb{R}$ denote the set of real numbers, $\mathbb{R}^{+}$the set of nonnegative real numbers, $\mathbb{R}^{n}$ the $n$-dimensional Euclidean space and $\mathbb{R}^{n \times m}$ the set of all $n \times m$ real matrices. For $a, b \in \mathbb{R}$, then, $a \vee v(a \wedge b)$ means the maximum (minimum) of $a$ and $b$. For a real square matrix $X$, the notation $X>0(X \geq 0, X<0$, $X \leq 0)$ means that $X$ is real symmetric and positive definite (positive semi-definite, negative definite, negative semi-definite, respectively); $\lambda(X)$ denotes the set of eigenvalues of $X ; \bar{\lambda}(X)$ and $\underline{\lambda}(X)$ denote the maximum and minimum eigenvalues of $X$, respectively. For $\tau>0, \mathcal{C}\left([-\tau, 0] ; \mathbb{R}^{n}\right)$ denotes the family of continuous functions $\varphi$ from $[-\tau, 0]$ to $\mathbb{R}^{n}$ with the norm $\|\varphi\|=$ $\sup _{-\tau<\vartheta \leq 0}|\varphi(\vartheta)|$. Let $\left(\Omega, \mathcal{F},\left\{\mathcal{F}_{t}\right\}_{t \geq 0}, P\right)$ be a complete probability space with a filtration $\left\{\mathcal{F}_{t}\right\}_{t \geq 0}$ satisfying the usual conditions (i.e., it is right continuous and $\mathcal{F}_{0}$ contains all $P$-pull sets); $\mathcal{C}_{\mathcal{F}_{0}}^{b}\left([-\tau, 0] ; \mathbb{R}^{n}\right)$ the family of all bounded, $\mathcal{F}_{0}$-measurable, $\mathcal{C}\left([-\tau, 0] ; \mathbb{R}^{n}\right)$-valued random variables; $\mathcal{C}^{2,1}\left(\mathbb{R}^{n} \times \mathbb{R}^{+} ; \mathbb{R}^{+}\right)$ the family of all nonnegative functions $V(x, t)$ on $\mathbb{R}^{n} \times \mathbb{R}^{+}$ which are continuously twice differentiable in $x$ and differentiable in $t$. If $x(t)$ is a continuous $\mathbb{R}^{n}$-valued stochastic process on $t \in[-\tau, \infty)$, let $x_{t}=\{x(t+\theta):-\tau \leq \theta \leq 0\}$ for $t \geq 0$, which is regarded as a $\mathcal{C}\left([-\tau, 0] ; \mathbb{R}^{n}\right)$-valued stochastic process. The mathematical expectation operator with respect to the given probability measure $P$ is denoted by $\mathbb{E}\{\cdot\}$.

\section{Problem Formulation AND Assumptions}

In [8], Hopfield brought forward a general class of neural networks, named Hopfield neural networks, which has been applied to various engineering fields. However, these applications are heavily dependent on the property of the stability of the equilibrium. The model of Hopfield neural networks can be expressed as follows:

$$
\dot{u}(t)=-A u(t)+B g(u(t))+J
$$

where $u(t)=\left(u_{1}(t), u_{2}(t), \ldots, u_{n}(t)\right)^{T} \in \mathbb{R}^{n}$ is the state vector associated with the neurons, $A=\operatorname{diag}\left(a_{1}, a_{2}, \ldots, a_{n}\right)>0$ is a positive diagonal matrix, $B=\left(b_{i j}\right)_{n \times n} \in \mathbb{R}^{n \times n}$ is the interconnection matrix, $g(u(t))=\left(g_{1}\left(u_{1}(t)\right), g_{2}\left(u_{2}(t)\right), \ldots, g_{n}\left(u_{n}(t)\right)\right)^{T}$ is the neuron activation function vector, $J=\left(J_{1}, J_{2}, \ldots, J_{n}\right)^{T}$ is a constant external input vector.

In recent years, Hopfield neural networks with time-varying delays have been widely investigated and several stability criteria have been obtained by many, see [5], [6], [10], for instances. The model of Hopfield neural networks with time-varying delays can be described by the following state equations:

$$
\dot{u}(t)=-A u(t)+B g(u(t-\tau(t)))+J
$$

where $\tau(t)>0$ is the transmission delay in (2) which is timevarying.

In this paper, the following assumptions are made.

(H1) There exists a positive diagonal matrix

$$
K=\operatorname{diag}\left(k_{1}, k_{2}, \ldots, k_{n}\right)>0,
$$

such that

$$
\left|g_{i}\left(u_{i}\right)-g_{i}\left(v_{i}\right)\right| \leq k_{i}\left|u_{i}-v_{i}\right| \quad \forall u_{i}, \quad v_{i} \in \mathbb{R}
$$

for $i=1,2, \ldots, n$.
(H2) $\tau(t)$ is bounded on $\mathbb{R}$, i.e., $0 \leq \tau(t) \leq \tau$, and is a differentiable function with $\dot{\tau}(t) \leq \zeta<1$, where $\tau, \zeta$ are constants.

In general, the activation functions are assumed to be bounded, differentiable, and monotonic increasing, such as the usual sigmoid functions which have been used in [1], [2], [8], [9], [11], [14]. However, in this brief, these restrictions on the activation functions $g_{i}$ are removed, which are only Lipschitz continuous (H1). Therefore, our hypothesis on the activation functions are more general than those in [1], [2], [8], [9], [11], [14].

As mentioned in Section I, it is reasonable to assume that the neural network (2) has only one equilibrium point, denoted by $u^{*}=\left(u_{1}^{*}, u_{2}^{*}, \ldots, u_{n}^{*}\right)^{T}$, which satisfies $-A u^{*}+B g\left(u^{*}\right)+J=$ 0 . By making a transformation $x(t)=u(t)-u^{*}$; then, (2) can be rewritten as

$$
\dot{x}(t)=-A x(t)+B f(x(t-\tau(t)))
$$

where $f(x(t-\tau(t)))=\left(f_{1}\left(x_{1}(t-\tau(t))\right), f_{2}\left(x_{2}(t-\right.\right.$ $\left.\tau(t))), \ldots, f_{n}\left(x_{n}(t-\tau(t))\right)\right)^{T}$ and $f_{i}\left(x_{i}(t-\tau(t))\right)=$ $g_{i}\left(x_{i}(t-\tau(t))+u_{i}^{*}\right)-g_{i}\left(u_{i}^{*}\right)$. Noting (3), it implies that $f$ satisfies

$$
\left|f_{i}\left(x_{i}\right)\right| \leq k_{i}\left|x_{i}\right|, \quad i=1,2, \ldots, n
$$

for all $x_{i} \in \mathbb{R}$.

In [18], a fuzzy dynamic TS model was proposed to represent local linear input/output relationships of nonlinear systems by using fuzzy IF-THEN rules. Recently, the ordinary TS fuzzy models have been extended to the case with time delay, and the stability has also been studied [4]. On the other hand, a neural network is often disturbed by environmental noises which affect the stability of the equilibrium. Motivated by these, we will generalize the ordinary TS fuzzy models to express a complex stochastic system whose consequent parts are a set of stochastic Hopfield neural networks with time-varying delays.

In this brief, a general class of SFVDHNNs, is discussed. As in [18], the model of SFVDHNN is composed of $r$ plant rules that can be described as follows:

\section{Plant Rule $i$ :}

$$
\begin{aligned}
& \operatorname{IF} \theta_{1}(t) \text { is } \eta_{1}^{i} \text { and } \cdots \text { and } \theta_{p}(t) \text { is } \eta_{p}^{i} \\
& \text { THEN } \\
& \begin{aligned}
d x(t) & =\left[-A_{i} x(t)+B_{i} f(x(t-\tau(t)))\right] d t \\
& +\sigma_{i}(x(t), x(t-\tau(t)), t) d w(t), \text { on } t \geq 0
\end{aligned} \\
& x(t)=\phi(t), \text { on } t \in[-\tau, 0] .
\end{aligned}
$$

where $i=1,2, \ldots, r, \eta_{j}^{i}$ is the fuzzy set, $\theta(t)=\left(\theta_{1}(t), \theta_{2}(t), \ldots, \theta_{p}(t)\right)^{t}$ is the premise variable vector, $r$ is the number of fuzzy IF-THEN rules. $w(t)=\left(w_{1}(t), w_{2}(t), \ldots, w_{m}(t)\right)^{T}$ is an $m$-dimensional Brownian motion defined on $\left(\Omega, \mathcal{F},\left\{\mathcal{F}_{t}\right\}_{t \geq 0}, P\right)$. Let $\sigma_{i}: \mathbb{R}^{n} \times \mathbb{R}^{n} \times \mathbb{R}^{+} \rightarrow \mathbb{R}^{n \times m}$, that is, $\sigma_{i}(x, y, t)=\left(\sigma_{j k}^{i}(x, y, t)\right)_{n \times m} \in \mathbb{R}^{n \times m}$. Throughout this paper, we assume that $\sigma_{i}(x, y, t)$ is locally Lipschitz continuous and satisfies the linear growth condition as well. Hence, it is known [15] that (6) with (7) has a unique global solution on $t \geq 0$ with the initial value $\phi \in \mathcal{C}_{\mathcal{F}_{0}}^{b}\left([-\tau, 0] ; \mathbb{R}^{n}\right)$. Moreover, we assume that $\sigma(0,0, t) \equiv 0$ such that (6) has an trivial solution $x(t, 0) \equiv 0$. 
The defuzzified output of system (6) is represented as follows:

$$
\begin{aligned}
d x(t)= & \sum_{i=1}^{r} h_{i}(\theta(t)) \\
& \times\left[\left(-A_{i} x(t)+B_{i} f(x(t-\tau(t)))\right) d t\right. \\
& \left.\quad+\sigma_{i}(x(t), x(t-\tau(t)), t) d w(t)\right]
\end{aligned}
$$

where

$$
h_{i}(\theta(t))=\frac{\nu_{i}(\theta(t))}{\sum_{j=1}^{r} \nu_{j}(\theta(t))} \quad \nu_{i}(\theta(t))=\prod_{j=1}^{p} \eta_{j}^{i}\left(\theta_{j}(t)\right)
$$

in which $\eta_{j}^{i}\left(\theta_{j}(t)\right)$ is the grade of membership of $\theta_{j}(t)$ in $\eta_{j}^{i}$. According to the theory of fuzzy sets, it is obvious that

$$
\nu_{i}(\theta(t)) \geq 0, \quad i=1,2, \ldots, r, \quad \sum_{j=1}^{r} \nu_{j}(\theta(t))>0
$$

for all $t$. Therefore, it implies

$$
h_{i}(\theta(t)) \geq 0, \quad i=1,2, \ldots, r, \quad \sum_{j=1}^{r} h_{j}(\theta(t))=1
$$

for all $t$.

Throughout this paper, we assume that all membership functions are continuous and piecewise continuously differentiable and the defuzzified model is also continuous. Clearly, based on the above discussion, (8) has a unique global solution on $t \geq 0$ through the initial value $x(\vartheta)=\phi(\vartheta)$ on $-\tau \leq \vartheta \leq 0$ in $\mathcal{C}_{\mathcal{F}_{0}}^{b}\left([-\tau, 0] ; \mathbb{R}^{n}\right)$, denoted by $x(t, \phi)$. And it is known that (8) has an equilibrium $x(t, 0) \equiv 0$.

Remark 1: By combining the extended version of the ordinary TS fuzzy models and the theory of delayed Hopfield neural networks, the model of SFVDHNN is proposed. A set of stochastic delayed Hopfield neural networks are used as the consequent parts of individual plant rules. The overall model of SFVDHNN is achieved by fuzzy "mixing" these stochastic delayed models. And the system dynamics is captured by these fuzzy implications which characterize local relation in the state space. In the same way, some similar stochastic fuzzy neural networks can also be established by using different types neural networks, such as cellular neural networks, bidirectional associative memory (BAM) neural networks, etc.

\section{STABILITY CRITERION FOR SFVDHNN}

Definition 1: For SFVDHNN (8) and every $\phi \in$ $\mathcal{C}_{\mathcal{F}_{0}}^{b}\left([-\tau, 0] ; \mathbb{R}^{n}\right)$, the trivial solution is globally exponentially stable in the mean square if there exist positive scalars $\alpha>0$ and $\beta>0$ such that

$$
\mathbb{E}\|x(t ; \phi)\|^{2} \leq \alpha e^{-\beta t} \mathbb{E}\|\phi\|^{2} .
$$

For stochastic systems, Itô's formula has an important role in the analysis of stochastic systems. The details can be found in [15] and omitted here. In the sequel, the main stability theorem and its proof will be given for determining the global exponential stability in the mean square of SFVDHNN.

Theorem 1: Let the activation function $g$ and the time delay $\tau(t)$ satisfy (H1) and (H2). Suppose that there exist some real matrices $C_{1} \geq 0, C_{2} \geq 0, C_{3} \geq 0$ and $P>0$, such that for all $i, j=1,2, \ldots, r$

$$
\begin{aligned}
\operatorname{trace} & {\left[\sigma_{i}^{T}(x(t), x(t-\tau(t)), t) P \sigma_{j}(x(t), x(t-\tau(t)), t)\right] } \\
\leq & x^{T}(t) C_{1} x(t)+x^{T}(t-\tau(t)) C_{2} x(t-\tau(t)) \\
& +f^{T}(x(t-\tau(t))) C_{3} f(x(t-\tau(t))) .
\end{aligned}
$$

The SFVDHNN (8) is globally exponentially stable in the mean square, if there exist a matrix $Q_{1}>0$ and a diagonal matrix $Q_{2}>0$, such that the following condition holds for $i=$ $1,2, \ldots, r$ :

$$
\Pi_{i}=\left(\begin{array}{ccc}
\Upsilon_{i} & P B_{i} & 0 \\
B_{i}^{T} P & -\eta Q_{2}+C_{3} & 0 \\
0 & 0 & -\eta Q_{1}+C_{2}
\end{array}\right)<0
$$

where $\Upsilon_{i}=-P A_{i}-A_{i} P+Q_{1}+K Q_{2} K+C_{1}, \eta=1-\zeta$.

Proof: Since $\Pi_{i}<0$, then it can guarantee that there is a sufficiently small scalar $\beta>0$ such that

$$
\Sigma_{i}=\left(\begin{array}{ccc}
\Xi_{i} & P B_{i} & 0 \\
B_{i}^{T} P & -\eta Q_{2}+C_{3} & 0 \\
0 & 0 & -\eta Q_{1}+C_{2}
\end{array}\right) \leq 0
$$

where $\Xi_{i}=\beta P-P A_{i}-A_{i} P+e^{\beta \tau} Q_{1}+e^{\beta \tau} K Q_{2} K+C_{1}$. In fact, it is obvious that $\Delta=\beta P+\left(e^{\beta \tau}-1\right) Q_{1}+\left(e^{\beta \tau}-\right.$ 1) $K Q_{2} K \geq 0$ for all $\beta \geq 0$, and equality holds when $\beta=0$. Moreover, we have $\Xi_{i}=\Upsilon_{i}+\Delta$, hence there exists $\beta>0$ such that $\Sigma_{i} \leq 0$.

Define a Lyapunov-Krasovskii functional candidate $V(x(t), t) \in \mathcal{C}^{2,1}\left(\mathbb{R}^{n} \times \mathbb{R}^{+} ; \mathbb{R}^{+}\right)$by

$$
\begin{aligned}
V(x(t), t)= & e^{\beta t} x^{T}(t) P x(t) \\
& +\int_{t-\tau(t)}^{t} e^{\beta(s+\tau)} x^{T}(s) Q_{1} x(s) d s \\
& +\int_{t-\tau(t)}^{t} e^{\beta(s+\tau)} f^{T}(x(s)) Q_{2} f(x(s)) d s .
\end{aligned}
$$

By Itô's formula, the stochastic derivative of $V(x(t), t)$ along (8) can be obtained

$$
\begin{aligned}
d V(x(t), t) \leq \sum_{i=1}^{r} & h_{i}(\theta(t)) e^{\beta t} \\
\times\{ & \beta x^{T}(t) P x(t)+e^{\beta \tau} x^{T}(t) Q_{1} x(t) \\
& \quad(1-\zeta) x^{T}(t-\tau(t)) Q_{1} x(t-\tau(t)) \\
& +e^{\beta \tau} x^{T}(t) K Q_{2} K x(t)-(1-\zeta) f^{T} \\
& \times\left(x(t-\tau(t)) Q_{2} f(x(t-\tau(t)))\right. \\
& +x^{T}(t)\left(-P A_{i}-A_{i} P\right) x(t) \\
& +x^{T}(t) P B_{i} f(x(t-\tau(t))) \\
& +f^{T}(x(t-\tau(t))) B_{i}^{T} P x(t) \\
& +x^{T}(t) C_{1} x(t) \\
& +x^{T}(t-\tau(t)) C_{2} x(t-\tau(t)) \\
& \left.+f^{T}(x(t-\tau(t))) C_{3} f(x(t-\tau(t)))\right\} d t \\
+ & M(t) \\
=e^{\beta t} & \sum_{i=1}^{r} h_{i}(\theta(t))\left(\begin{array}{c}
f(x(t) \\
x(t-\tau(t))
\end{array}\right) \\
\times & \left(\begin{array}{c}
x(t) \\
f(x(t-\tau(t)))
\end{array}\right) d t+M(t) \\
& (11)
\end{aligned}
$$

where

$$
\begin{aligned}
M(t)=e^{\beta t} \sum_{i=1}^{r} h_{i}(\theta(t)) & {\left[x^{T}(t) P \sigma_{i}(x(t), x(t-\tau(t)), t)\right.} \\
+ & \left.\sigma_{i}^{T}(x(t), x(t-\tau(t)), t) P x(t)\right] d w(t) .
\end{aligned}
$$


Since $\Sigma_{i} \leq 0$, hence it follows that

$$
\mathbb{E} V(x(t), t) \leq \mathbb{E} V(0) .
$$

Now, from (10), we obtain

$\mathbb{E} V(0) \leq\left\{\bar{\lambda}(P)+\frac{e^{\beta \tau}-1}{\beta}\left[\bar{\lambda}\left(Q_{1}\right)+\bar{\lambda}\left(K Q_{2} K\right)\right]\right\} \mathbb{E}\|\phi\|^{2}$.

On the other hand, from the definition of $V(x(t), t)$, one obtains

$$
\mathbb{E} V(x(t), t) \geq \underline{\lambda}(P) e^{\beta t} \mathbb{E}\|x(t)\|^{2} .
$$

Combining (12) and (13), it can be easily obtained that

$$
\mathbb{E}\|x(t, \phi)\|^{2} \leq \alpha e^{-\beta t} \mathbb{E}\|\phi\|^{2},
$$

where

$$
\alpha=\frac{\bar{\lambda}(P)+\frac{e^{\beta \tau}-1}{\beta}\left[\bar{\lambda}\left(Q_{1}\right)+\bar{\lambda}\left(K Q_{2} K\right)\right]}{\underline{\lambda}(P)} .
$$

That is, SFVDHNN (8) is globally exponentially stable in the mean square. This completes the proof.

Remark 2: In this brief, by constructing one appropriate Lyapunov-Krasovskii functional and using Itô's formula, sufficient condition for SFVDHNN is given to guarantee the global exponential stability in the mean square of the trivial solution. It can be seen that the stability criterion is formulated in terms of LMIs which can be easily checked in practice, so it possesses important leading significance in the design and applications of SFVDHNN. In addition, the approaches adopted in this paper can also be used to the stability analysis for other stochastic fuzzy neural networks with time-varying delay, such as stochastic fuzzy cellular neural networks and stochastic fuzzy BAM neural networks, etc.

Remark 3: When $r=1$, (8) degenerates into the stochastic Hopfield neural networks with time-varying delay. Likewise, one sufficient condition on global exponential stability in the mean square is given for the stochastic Hopfield neural networks with time-varying delay. Recently, in [1], [2], [11], [14], some stability conditions were derived for the stochastic Hopfield neural networks with constant delay. In those papers, the activation functions $g_{i}$ were assumed to be nondecreasing and $\left|g_{i}(u)\right| \leq 1 \wedge \beta_{i}|u|$ for all $u \in \mathbb{R}$, the connection weights were required to satisfy $b_{i}=\sum_{j=1}^{n}\left|a_{i j}\right|, 1 \leq i \leq n$ or even to be symmetric. It is known that these assumptions in [1], [2], [11], [14] are very restrictive and limit their applications. Nevertheless, the above restrictions on the activation functions and the connected weight matrix are removed in our paper. We only suppose that the activation function $g$ to be Lipschitz continuous (H1). Moreover, our stability criterion can take into account the sign of entries in the connected matrix, which illustrates the differences between the excitatory and inhibitory effects. Hence, the result obtained in our paper is less conservative and less restrictive than the previous works.

\section{ILLUSTRATIVE EXAMPLE}

Here, we will present an example to illustrate the results developed.
Let $r=2$. Consider the following plant rules of a SFVDHNN:

\section{Plant Rule $i$ :}

$$
\begin{aligned}
& \operatorname{IF} \theta_{1}(t) \text { is } \eta_{1}^{i} \text { and } \ldots \text { and } \theta_{p}(t) \text { is } \eta_{p}^{i}, \text { THEN } \\
& \begin{aligned}
d x(t)= & {\left[-A_{i} x(t)+B_{i} f(x(t-\tau(t)))\right] d t } \\
& +\sigma_{i}(x(t), x(t-\tau(t)), t) d w(t), \quad \text { for } i=1,2
\end{aligned}
\end{aligned}
$$

where $\eta_{j}^{i}$ is a fuzzy set, $\theta(t)=\left(\theta_{1}(t), \ldots, \theta_{p}(t)\right)^{T}$ is the premise variable vector, $w(t)$ is a scalar Brownian motion and

$$
\begin{aligned}
f_{i}\left(x_{i}\right) & \equiv \frac{1}{2}\left[\left|x_{i}+1\right|-\left|x_{i}-1\right|\right] \\
\tau(t) & =\frac{1}{2} \sin t+\frac{1}{2} \\
\sigma_{1}(x, y, t) & =\left(0.5 x_{1}(t-\tau(t)), 0.4 x_{2}(t-\tau(t))\right)^{T} \\
\sigma_{2}(x, y, t) & =\left(0.2 x_{1}(t-\tau(t)), 0.3 x_{2}(t-\tau(t))\right)^{T} .
\end{aligned}
$$

Obviously, Assumptions (H1) and (H2) are satisfied with $K=$ $\operatorname{diag}(1,1)$ and $\tau=1, \zeta=(1 / 2)$, respectively. Thus

trace $\left(\sigma_{i}^{T}(x, y, t) \sigma_{j}(x, y, t)\right)$

$$
\leq 0.25 x_{1}^{2}(t-\tau(t))+0.16 x_{2}^{2}(t-\tau(t))
$$

for all $i, j=1,2$.

The SFVDHNN can be described by

$$
\begin{aligned}
d x(t)= & \sum_{i=1}^{2} h_{i}(\theta(t))\left[\left(-A_{i} x(t)\right.\right. \\
& \left.+B_{i} f(x(t-\tau(t)))\right) d t \\
& \left.+\sigma_{i}(x(t), x(t-\tau(t)), t) d w(t)\right] .
\end{aligned}
$$

Now we let

$$
\begin{array}{ll}
A_{1}=\left(\begin{array}{ll}
6 & 0 \\
0 & 4
\end{array}\right) & B_{1}=\left(\begin{array}{ll}
1 & -2 \\
0 & -1
\end{array}\right) \\
A_{2}=\left(\begin{array}{ll}
5 & 0 \\
0 & 6
\end{array}\right) & B_{2}=\left(\begin{array}{cc}
2 & -1 \\
1 & 2
\end{array}\right) .
\end{array}
$$

Then, there exist $C_{1}=C_{3}=0, C_{2}=\operatorname{diag}(0.25,0.16), P=$ $\operatorname{diag}(1,1)>0$

$$
Q_{1}=\left(\begin{array}{ll}
2 & 1 \\
1 & 1
\end{array}\right)>0 \text { and } Q_{2}=\left(\begin{array}{ll}
2 & 0 \\
0 & 2
\end{array}\right)>0
$$

such that the matrix $\Pi_{i}$ defined in Theorem 1 is negative definite for $i=1,2$. That is, the conditions of Theorem 1 are satisfied. Therefore, the above SFVDHNN is globally exponentially stable in the mean square.

\section{CONCLUSION}

In this brief, a general class of SFVDHNN has been studied. Firstly, the model of SFVDHNN has been proposed by further extending the ordinary TS fuzzy models, in which a set of stochastic delayed Hopfield neural networks constitute its consequent parts of individual plant rules. Secondly, the global exponential stability in the mean square has been investigated for SFVDHNN based on the Lyapunov-Krasovskii approach. One stability criterion has been derived in this paper. The stability condition is expressed in terms of the solutions to a set of LMIs, which can be solved numerically and very effectively by using 
for example the interior-point method. Furthermore, our result is allowed to have a wider choice for the activation functions, including the usual sigmoid functions and the piecewise-linear functions.

\section{REFERENCES}

[1] S. Blythe, X. R. Mao, and X. X. Liao, "Stability of stochastic delay neural networks," J. Franklin Inst., vol. 338, pp. 481-495, 2001.

[2] S. Blythe, X. R. Mao, and A. Shah, "Razumikhin-type theorems on stability of stochastic neural networks with delays," Stochast. Anal. Appl., vol. 19 , no. 1, pp. 85-101, 2001.

[3] S. Boyd, L. E. Ghaoui, E. Feron, and V. Balakrishnan, Linear Matrix Inequalities in System and Control Theory. Philadelphia, PA: SIAM, 1994.

[4] Y. Y. Cao and P. M. Frank, "Analysis and synthesis of nonlinear timedelay systems via fuzzy control approach," IEEE Trans. Fuzzy Syst., vol. 8, no. 2, pp. 200-211, Feb. 2000.

[5] J. D. Cao and J. Wang, "Global asymptotic stability of a general class of recurrent neural networks with time-varying delays," IEEE Trans. Circuits Syst. I, Fundam. Theory Appl., vol. 50, no. 1, pp. 34-44, Jan. 2003.

[6] P. van den Driessche and X. Zou, "Global attractivity in delayed Hopfield neural network models," SIAM J. Appl. Math., vol. 58, no. 6, pp. 1878-1890, 1998.

[7] P. Gahinet, A. Nemirovsky, A. J. Laub, and M. Chilali, LMI Control Toolbox: For Use With Matlab. Natick, MA: The Math Works, Inc, 1995.
[8] J. J. Hopfield, "Neural networks and physical systems with emergent collect computational abilities," Proc. Nat. Acad. Sci. USA, vol. 79, no. 2, pp. 2554-2558, 1982.

[9] properties like those of two-state neurons," Proc. Nat. Acad. Sci. USA, vol. 81, pp. 3088-3092, 1984.

[10] C. Hou and J. Qian, "Stability analysis for neural dynamics with timevarying delays," IEEE Trans. Neural Netw., vol. 9, no. 1, pp. 221-223, Feb. 1998.

[11] S. G. Hu, X. X. Liao, and X. R. Mao, "Stochastic Hopfield neural networks," J. Phys. A: Math. Gen, vol. 36, pp. 2235-2249, 2003.

[12] G. Joya, M. A. Atencia, and F. Sandoval, "Hopfield neural networks for optimization: Study of the different dynamics," Neurocomp., vol. 43, pp. 219-237, 2002.

[13] W. J. Li and T. Lee, "Hopfield neural networks for affine invariant matching," IEEE Trans. Neural Netw., vol. 12, no. 6, pp. 1400-1410, Dec. 2001.

[14] X. X. Liao and X. R. Mao, "Exponential stability and instability of stochastic neural networks," Stochast. Anal. Appl., vol. 14, no. 2, pp. $165-185,1996$.

[15] X. R. Mao, Stochastic Differential Equations and Applications. Chichester, U.K.: Horwood, 1997.

[16] C. M. Marcus and R. M. Westervelt, "Stability of analog neural networks with delays," Phys. Rev. A, vol. 39, no. 1, pp. 347-359, 1989.

[17] Y. Nesterov and A. Nemirovsky, Interior Point Polynomial Methods in Convex Programming. Philadelphia, PA: SIAM, 1994.

[18] T. Takagi and M. Sugeno, "Fuzzy identification of systems and its applications to modeling and control," IEEE Trans. Syst. Man, Cybern., vol. SMC-15, no. 1, pp. 116-132, Jan. 1985.

[19] S. S. Young, P. D. Scott, and N. M. Nasrabadi, "Object recognition using multilayer Hopfield neural network," IEEE Trans. Image Process., vol. 6, no. 3, pp. 357-372, 1997. 